Varia

\section{Komplikationen und Revisionen nach elektiven handchirurgischen Eingriffen selten}

Goodman AD et al. Thirty-Day Reoperation and/ or Admission After Elective Hand Surgery in Adults: A 10-Year Review. J Hand Surg Am 2018; 43: 383.e1 - 383.e7

Zur Häufigkeit akuter Komplikationen nach elektiven handchirurgischen Eingriffen ist wenig bekannt und die spärlichen vorhandenen Daten stammen meist aus Datenbanken, die administrativen Zwecken dienen. Daraus lassen sich aber keine Informationen zu möglichen Risikofaktoren für derartige Komplikationen ableiten, daher haben nun USamerikanische Mediziner Krankenakten ihrer Praxis retrospektiv ausgewertet.

Dazu haben Avi Goodman und seine Kollegen für den Zeitraum Februar 2006 bis Januar 2016 insgesamt mehr als 18000 elektive handchirurgische Operationen bei erwachsenen Patienten beurteilt. Mehr als die Hälfte dieser Eingriffe (57,6\%) waren Behandlungen eines Karpaltunnelsyndroms und/oder einer Tendovaginosis stenosans.

Als Indikatoren für relevante Komplikationen verwendeten die Mediziner dabei die Notwendigkeit ungeplanter Revisionen bzw. stationärer Aufnahmen innerhalb von 30 Tagen nach dem primären Eingriff. Bei den so ermittelten Patienten suchten sie nach der Art der Komplikationen und möglicherweise damit verbundenen Faktoren.

Die Auswertung ergab 27 akute Komplikationen innerhalb von 30 Tagen nach der Primäroperation. Das entspricht einer Inzidenz von insgesamt 0,15\%; mit geringeren Zahlen nach einfacheren Eingriffen wie der Spaltung des Retinaculum flexorum oder eines Ringbands (0,09$0,10 \%$ ) und höheren nach umfangreicheren Eingriffen z.B. am Handgelenk $(0,74 \%)$.
Infektionen machten mehr als die Hälfte der Komplikationen aus ( $n=17)$; nachgewiesene Erreger umfassten einen Methicillin-empfindlichen Staphylococcus aureus $(n=9)$, einen Methicillin-resistenten Staphylococcus aureus $(n=3)$ und einen Koagulase-negativen Staphylococcus sp. $(n=1)$. Die restlichen 10 Komplikationen umfassten Übelkeit und Erbrechen, ambulant nicht beherrschbare Schmerzen, Wunddehiszenzen, Nachblutungen und neurologische Irritationen. Bei 11 der Patienten mit Komplikationen lag eine Grunderkrankung mit Immunsuppression vor (Diabetes mellitus, rheumatoide Arthritis unter Therapie, Zustand nach Splenektomie).

Nach der Clavien-Dindo-Klassifikation handelte es sich um eine Komplikation Grad I (ungeplante stationäre Aufnahme zur Schmerztherapie), 4 Komplikationen Grad II (intravenöse Behandlung mit Antibiotika ohne Revision) und 22 Komplikationen Grad III (operative Revision erforderlich). Lebensbedrohliche oder tödliche Komplikationen (Grad IV oder V) traten nicht auf.

Die Probleme wurden in ihrer Mehrzahl zwischen dem 8. und 14. postoperativen Tag diagnostiziert ( $n=16 ; 59,2 \%$ ), etwas mehr als ein Viertel der Komplikationen trat bis zum 7. postoperativen Tag auf ( $n=8 ; 29,6 \%$ ), und nur etwa jede 10 . zwischen Tag 14 und Tag 21. Nach der 3. Woche postoperativ waren bis Tag 30 keine Probleme mehr zu verzeichnen. Spätkomplikationen nach Tag 30, z.B. anhaltende Funktionseinschränkungen oder ein komplexes regionales Schmerzsyndrom, waren in den Unterlagen allerdings nicht erfasst.
FAZIT

Demnach scheint das Komplikationsrisiko nach elektiven handchirurgischen Eingriffen mit 15 Fällen auf 10000 Operationen relativ gering, so die Autoren. Dabei scheint das Risiko v. a. bei Patienten mit Immunsuppression und nach komplexeren Eingriffen erhöht. Allerdings können ihre Daten nicht verallgemeinert werden, meinen Goodman et al. abschließend: Die Beschränkung auf elektive Eingriffe schließt Probleme nach Unfällen, Tierbissen und Infektionen als primäre OP-Indikation weitgehend aus.

Dr. Elke Ruchalla, Bad Dürrheim 